\title{
NOVOS XENACANTHIDAE (CHONDRICHTHYES, ELASMOBRANCHII) DA BASE DO MEMBRO TAQUARAL, FORMAÇÃO IRATI, PERMIANO DA BACIA DO PARANÁ
}

\author{
Artur CHAHUD \\ Setembrino PETRI
}

\begin{abstract}
RESUMO
A fácies arenosa do Membro Taquaral da Formação Irati é conhecida pela grande concentração de fósseis de Chondrichthyes, porém apenas uma espécie de Xenacanthiformes era conhecida, Xenacanthus albuquerquei. Pequenos dentes de Xenacanthidae, presentes na fácies arenosa da base do Membro Taquaral da Formação Irati (Eopermiano - Artinskiano), provenientes de afloramento do Sítio Santa Maria, município de Rio Claro, Estado de São Paulo, são aqui descritos. Foram identificadas duas espécies distintas: a primeira como pertencente ao gênero Xenacanthus e a outra como Xenacanthidae indeterminado.
\end{abstract}

Palavras-chave: Chondrichthyes, Formação Irati, Xenacanthidae, Membro Taquaral

\section{ABSTRACT}

The sandy facies at the base of the Taquaral Member, Irati Formation (early Permian, Artinskian), is noteworthy for its abundance of chondrichthyan fossils. However, to date just one species of Xenacanthiformes, Xenacanthus albuquerquei, has been published. Small teeth in this facies in an outcrop on the Santa Maria Homestead, Rio Claro Municipality, State of São Paulo, Brazil, are here assigned to two taxa, one to Xenacanthus $\mathrm{sp}$. and the other as an indeterminate Xenacanthidae.

Keywords: Chondrichthyes, Irati Formation, Xenacanthidae, Taquaral Member

\section{INTRODUÇÃO}

Durante o Paleozóico e início do Mesozóico, os rios e regiões costeiras eram habitados por diversas espécies de Chondrichthyes, entre elas os tubarões Xenacanthiformes. Os primeiros fósseis de Xenacanthiformes foram descobertos na Europa, no decorrer da década de 1830, principalmente na Inglaterra e Alemanha e, na década de 1850, nos Estados Unidos.

Os tubarões xenacantos eram peixes de tamanho médio (3,5 $\mathrm{m}$ de comprimento), de corpo fino e alongado, facilmente distinguíveis de outros grupos de elasmobrânquios (HAMPE 2003). A maior parte das espécies habitava rios e lagos de água doce, porém algumas, principalmente no Eocarbonífero, preferiam salinidades maiores como estuários e ambientes costeiros (BAIRD 1978, PARRISH 1978, JANVIER 1996, JOHNSON 2005a, b). Não é incomum encontrar dentes e espinhos de espécies de água doce retrabalhados em depósitos marinhos costeiros, de alta energia ou de origem deltaica (MOY-THOMAS \& MILES 1971, LONG 1995, NELSON 2006).

Entre os fósseis preservados deste grupo de Chondrichthyes, estão dentes, espinhos, escamas e raros indivíduos articulados. Dentes e espinhos, por serem mais comumente preservados, são as principais estruturas de identificação e estudo sistemático (HAMPE 2003).

Atualmente, são reconhecidas duas famílias de Xenacanthiformes; Diplodoselachidae e Xenacanthidae (HAMPE 2003), ambas encontradas na Bacia do Paraná, com gêneros e espécies endêmicos (RAGONHA 1978, 1984; RICHTER 2005).

A referência mais antiga na literatura de Xenacanthiformes na Bacia do Paraná é atribuída à espécie Xenacanthus albuquerquei, também observada na Formação Pedra do Fogo (SILVA SANTOS 1946, RAGONHA 1978, CHAHUD \& PETRI 2008). Esta era, até o momento, a única espécie de 
Xenacanthiformes da base do Membro Taquaral, porém pequenos exemplares atribuídos a família Xenacanthidae foram recentemente descobertos. O objetivo desta contribuição é apresentar dois novos espécimes de pequenos dentes, representados por Xenacanthiformes provenientes da fácies arenosa do Membro Taquaral.

\section{MATERIAL E MÉTODOS}

O estudo foi realizado em um afloramento do Sítio Santa Maria, município de Rio Claro, Estado de São Paulo (Figura 1). Todos os fósseis provêm de uma camada horizontal, pouco intemperizada, de 9,5 cm de espessura (Figura 2), exposta em uma área de 20 por $7 \mathrm{~m}$, na margem esquerda (SW) do rio Cabeça (UTM: 23K 0227055/7517325), a aproximadamente $850 \mathrm{~m}$ NNW da entrada do sítio (Figura 1).

Todos os espécimes estão registrados e depositados na coleção fóssil (GP/2T) do Laboratório de Paleontologia Sistemática (LPS) do Instituto de Geociências da Universidade de São Paulo (IGc-USP).

Imagens digitais foram obtidas a partir de um Omega Megascan - ACCU 6000 Scanner e uma máquina fotográfica analógica SONY $3 \mathrm{CCD}$, processadas em um analisador de imagem da LEICA do Laboratório de Petrografia Sedimentar do Instituto de Geociências da Universidade de São Paulo (IGc-USP).

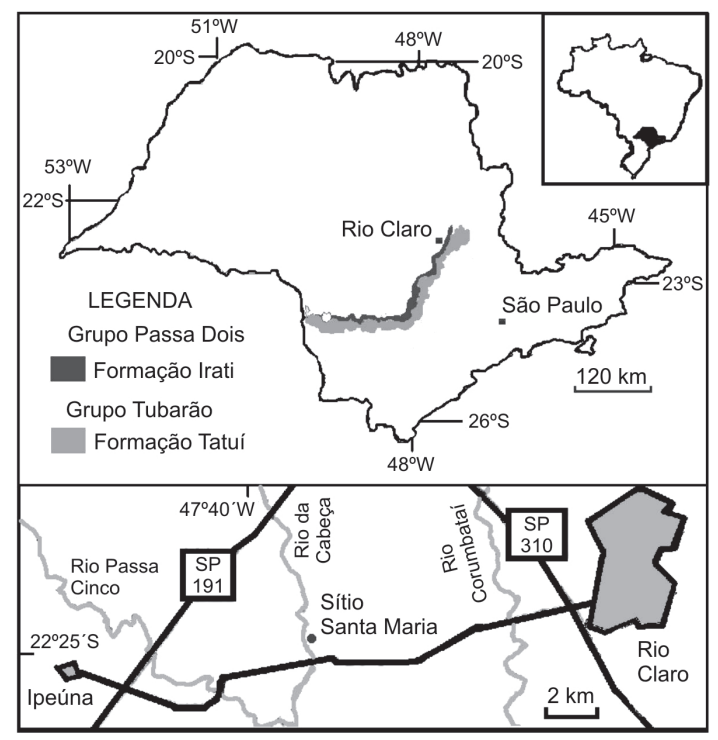

FIGURA 1 - Mapa do Estado de São Paulo destacando as faixas de ocorrência das formações Tatuí e Irati. Abaixo, mapa local destacando as principais rodovias de acesso ao Sítio Santa Maria.

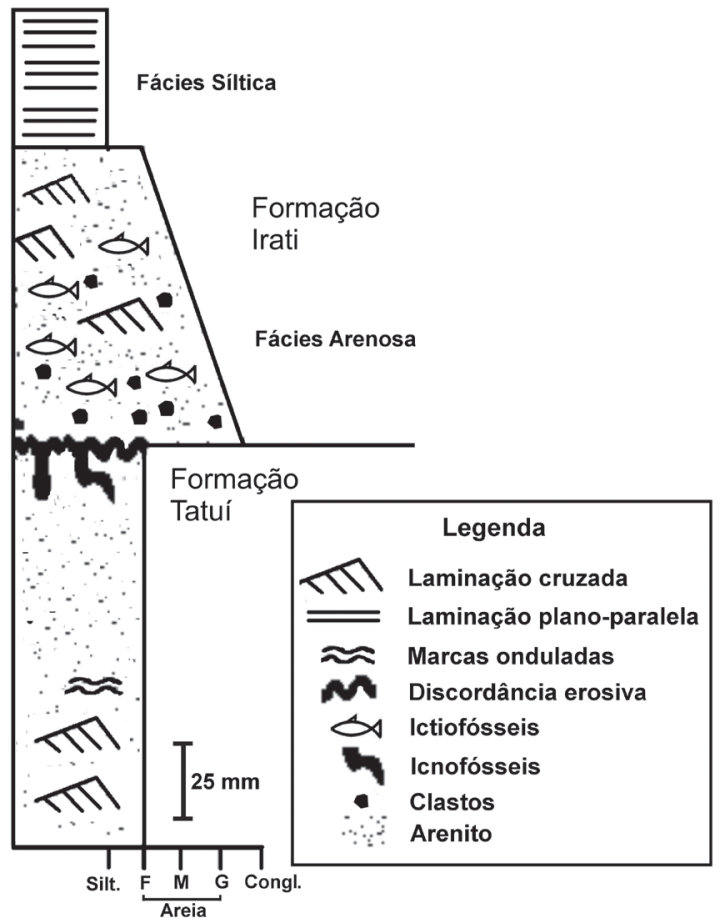

FIGURA 2 - Seção do afloramento no Sítio Santa Maria, município de Rio Claro.

\section{PALEONTOLOGIA SISTEMÁTICA}

Classe CHONDRICHTHYES Huxley. 1880 Subclasse ELASMOBRANCHII Bonaparte, 1838 Ordem XENACANTHIFORMES Berg, 1940 Família XENACANTHIDAE Fritsch, 1889 Xenacanthus sp.

Material - GP/2T-215. Dente isolado fixo na matriz (Figura 3).

Descrição - $\mathrm{O}$ dente apresenta muitos traços de abrasão por toda a sua superfície, mas de modo homogêneo, com todas as bordas laterais e as cúspides arredondadas. Possui duas cúspides laterais que se destacam, desenvolvendo-se paralelamente entre si, voltadas para a face labial da base.

A base é triangular e pouco espessa, com grande botão apical (ou botão coronal) ocupando todo o espaçamento entre as cúspides e a região centro-labial. Entre o botão apical e a borda da base ocorrem pequenos foramens nutritivos. A altura da cúspide maior é $1,6 \mathrm{~mm}$ e da menor $1,5 \mathrm{~mm}$ e a base possui $1,3 \mathrm{~mm}$ de largura. $\mathrm{O}$ botão apical tem $0,6 \mathrm{~mm}$ de largura e $0,9 \mathrm{~mm}$ de comprimento, medido na distância lingual-labial. As cúspides localizam-se mais ou menos na mesma região da face 


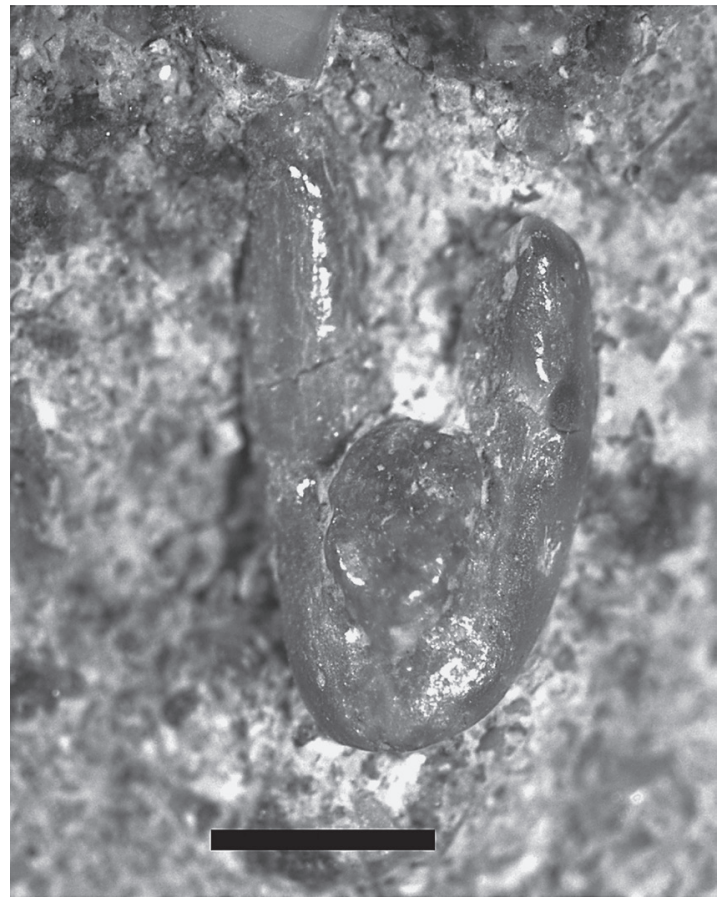

FIGURA 3 - Dente de Xenacanthus sp. GP/2T-215. Escala $1 \mathrm{~mm}$.

labial e não há cúspide mediana; são ligeiramente arredondadas em corte transversal, com um pequeno achatamento lábio-lingual; apesar da abrasão, são originalmente lisas, a menor, com algumas fraturas. $\mathrm{O}$ ângulo lateral entre a coroa e a base é superior a $130^{\circ}$.

Discussão - As cúspides lisas, aproximadamente circulares em vista transversal, o grande botão apical e os foramens nutritivos próximos da base, são características diagnósticas do gênero Xenacanthus (HAMPE 2003). Como apenas um exemplar foi coletado com as características aqui descritas, não foi possível a identificação em nível específico.

As cúspides paralelas entre si e com grande ângulo $\left(>\right.$ que $\left.120^{\circ}\right)$ em relação à base são características encontradas em três espécies do gênero Xenacanthus do Hemisfério Norte; X. laevissimus, $X$. elegans e X. tenuis. Porém, o exemplar estudado difere por não possuir cúspide intermediária e nem arqueamento nas cúspides maiores, comuns nas espécies do Hemisfério Norte.

Entre as espécies brasileiras, a que mais se assemelha é Xenacanthus pricei (WÜRDIG MACIEL 1975) do topo da Formação Irati do Rio Grande do Sul. A diferença entre ambas refere-se ao tamanho entre as cúspides principais divergentes, maior em X. pricei, além da ausência de cristas muito finas nas cúspides, que ocorrem na face superior de X. pricei (WÜRDIG - MACIEL 1975).
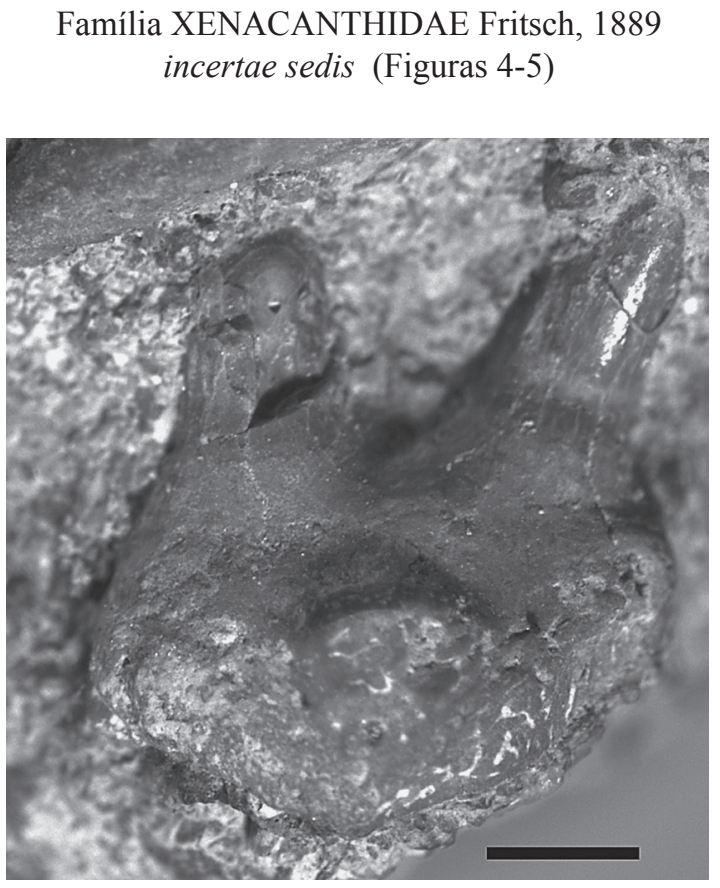

FIGURA 4 - Espécime GP/2T-216 de Xenacanthidae preso a matriz rochosa. Escala $1 \mathrm{~mm}$.

Material - GP/2T-216 (Figura 4) e GP/2T-217 (Figura 5). Dentes fragmentados.

Descrição de GP/2T-216- Coroa consistindo de duas cúspides divergentes, de tamanhos similares ( $1 \mathrm{~mm}$ de largura na parte mais espessa), sendo uma delas muito inclinada. Não existe evidência de que tenha havido uma cúspide intermediária. Apesar de fragmentadas, é possível inferir que as cúspides são alongadas. Observam-se estriações (possíveis cristas desgastadas) em uma das cúspides e não ocorre serrilhamento de nenhum tipo. A base é pouco espessa, com a face lingual voltada para baixo. Apesar do estado de preservação, é possível observar foramens nutritivos, um muito próximo do botão apical. O botão apical também apresenta quebras e é pequeno, totalmente isolado das cúspides, posicionado na face lingual. A base tem 3,4 mm de comprimento anteroposterior e $4,3 \mathrm{~mm}$ de altura. A cúspide maior mede aproximadamente 2,1 $\mathrm{mm}$ de altura e $0,9 \mathrm{~mm}$ de diâmetro. A cúspide menor tem aproximadamente $0,9 \mathrm{~mm}$ de diâmetro e 1,9 mm de altura. O botão apical tem $1 \mathrm{~mm}$ de comprimento máximo. 
Descrição de GP/2T-217 - dente composto por duas cúspides laterais de tamanhos ligeiramente diferentes, a maior com $0,9 \mathrm{~mm}$ de diâmetro e a menor com $0,7 \mathrm{~mm}$. Uma cúspide intermediária, situada entre as maiores, tem menos da metade do diâmetro da maior, em contato direto com as laterais das cúspides maiores, e está localizada obliquamente na face labial. Uma das laterais da base está preservada, sendo possível inferir que tem formato arredondado. Ocorrem

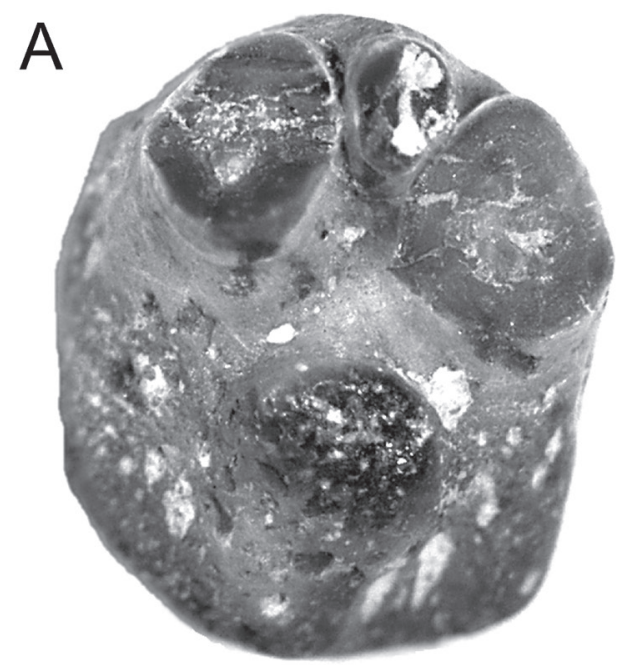

foramens nutritivos na parte superior lingual, próximos do botão apical e na região central na face inferior, onde verificou-se uma pequena depressão. A ausência do tubérculo basal talvez deva a uma quebra do formato arredondado na borda labial.

O botão apical tem contato com as cúspides, porém não chega a se posicionar na face labial e fica restrito à região central e parte da região lingual da base.

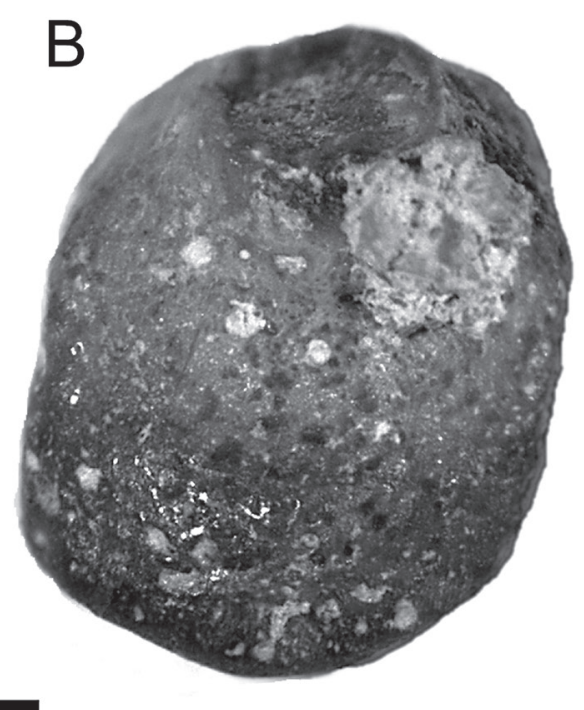

FIGURA 5 - Exemplar GP/2T-217. A) Vista oclusal; B) Vista inferior. Escala 1 mm.

Discussão - Os dois exemplares aparentemente pertencem à mesma espécie, pois as bases e o botão apical são muito semelhantes. A falta da cúspide intermediária no GP/2T-216 seria sugestiva de dentição heterodonte.

A classificação dos exemplares é incerta por serem os únicos e por estarem mal preservados, porém é possível sugerir afinidades e diferenças com alguns gêneros de Xenacanthiformes.

Os exemplares podem ser associados com segurança dentro dos Xenacanthidae, pelas cúspides circulares e sem evidências de bordos cortantes ou serrilhados, características distintas em Diplodoselache, Dicentrodus, Lebachacanthus, Orthacanthus e Hagenoselache (JOHNSON 1999, 2003; HAMPE 2003; HAMPE et al. 2006). A classificação em gênero conhecido ou novo é discutível, pois seria necessária análise de mais exemplares com as cúspides melhor conservadas.

A divergência das cúspides é comum em espécies de Orthacanthus e em Wurdgneria, porém em GP/2T-216, a ornamentação das cúspides, o botão apical e a base diferem, em tamanho e forma, dos gêneros citados acima.

A ornamentação por cristas longitudinais irregulares por toda a superfície, evidentes no GP/2T-216 e pouco claras no exemplar GP/2T-217, exclui a possibilidade de associação ao gênero $\mathrm{Xe}$ nacanthus, aproximando-os dos fósseis de Plicatodus e Triodus (HAMPE 2003)

Barbclabornia (JOHNSON 2003) é um gênero bicuspidado, assemelhando-se ao exemplar GP/2T-216, porém a base mais espessa e o grande botão apical o difere dos aqui descritos.

A morfologia dos dentes com duas cúspides de grande porte e tamanhos semelhantes o diferencia da espécie Xenacanthus albuquerquei, o Xenacanthiformes mais comum da unidade, no que diz respeito à morfologia das cúspides e do botão apical. Os exemplares aqui descritos diferem de Xenacanthus sp., aqui mencionado, pela grande diferença do botão apical, forma da base e disposição das cúspides. 


\section{CONSIDERAÇÕES PALEOAMBIENTAIS}

O Membro Taquaral é interpretado como de paleoambiente de salinidade variável, ora mais doce (MUSSA et al. 1980), ora com salinidade, mas com influência continental por rios (CHAHUD 2007). A fácies arenosa basal do Membro Taquaral foi gerada em condições de alta energia, por isso os fósseis estão muitos desgastados e fragmentados. Por esta razão uma correta interpretação paleoambiental não é possível, mas algumas hipóteses podem ser propostas.

Xenacanthus albuquerquei é o segundo Chondrichthyes mais abundante, sendo superado apenas pelo petalodonte Itapyrodus punctatus (RAGONHA 1978, CHAHUD 2007). O estado de preservação destes fósseis é variado, com exemplares muito bem preservados e outros muito desgastados, sugestivos de mistura temporal e, portanto, predominantemente parautóctone ou autóctone.

Os novos exemplares sofreram desgaste e são muito raros, sendo observados apenas no Sítio Santa Maria, limite dos municípios de Rio Claro e Ipeúna. Provavelmente são alóctones, transportados por rios junto com tetrápodes maiores (CHAHUD \& PETRI 2010), também presentes no depósito.

\section{CONCLUSÕES}

Os novos dentes de Xenacanthidae do Membro Taquaral são representados por, pelo menos, duas espécies. A primeira espécie pode ser atribuída ao gênero Xenacanthus e é similar a $X$. pricei do topo da Formação Irati do Estado do Rio Grande do Sul, porém seriam necessários mais exemplares para a confirmação. A segunda é de classificação incerta devido ao desgaste dos exemplares coletados.

Em geral os fósseis de Xenacanthidae são muito raros e estão desgastados sugerindo que sejam alóctones ao depósito, ao contrário de $X$. albuquerquei, que seria autóctone ou parautóctone, devido à presença abundante.

\section{AGRADECIMENTOS}

Os autores expressam seu agradecimento ao Professor Dr. Thomas Rich Fairchild, pelo apoio e auxílio dados em vários momentos durante esta pesquisa, aos proprietários do Sítio Santa Maria no município de Rio Claro, Senhor Luis e Senhora Bernardete, onde foi coletado o material, ao Departamento de Geologia Sedimentar e Ambiental que permitiu que os trabalhos de preparação dos fósseis fossem realizados em seus laboratórios. Por último um agradecimento especial a CAPES e FAPESP pelo apoio financeiro.

\section{REFERÊNCIAS BIBLIOGRÁFICAS}

BAIRD, O. 1978. Studies on Carboniferous freshwater fishes. American Museum Novitates, 1641:1-22.

CHAHUD, A. 2007. Paleontologia de vertebrados da transição entre os grupos Tubarão e Passa Dois no Centro-Leste do Estado de São Paulo. Instituto de Geociências, Universidade de São Paulo, São Paulo, Dissertação de Mestrado. 172 p.

CHAHUD, A.; S. PETRI. 2008. Chondrichthyes no Membro Taquaral, base da Formação Irati, no centro-leste do Estado de São Paulo, Brasil. Revista de Geologia 21: 169 - 179.

CHAHUD, A.; PETRI, S. 2010. Anfíbio e Paleonisciformes da Porção Basal do Membro Taquaral, Formação Irati (Permiano), Estado de São Paulo, Brasil. Geologia USP, Série Cientifica, 10(1): 29-37.

HAMPE, O. 2003. Revision of the Xenacanthida (Chondrichthyes: Elasmobranchii) from the Carboniferous of the British Isles. Transaction of the Royal Society of Edimburg: Earth Sciences, 93: 191-237.

HAMPE, O.; JOHNSON, G. D.; TURNER. S. 2006. Dicentrodus (Chondrichthyes: Xenacanthida) from the Early Carboniferous (Visean: upper St Louis Formation) of Iowa, USA. Geological Magazine, 143 (4): 545-549.

JANVIER, P. 1996. Early Vertebrates. Oxford Science Publications, 393p.

JOHNSON. G. 1999. Dentitions of Late Palaeozoic Orthacanthus species and new species of ?Xenacanthus (Chondrichthyes: Xenacanthiformes) from North América. Acta Geologica Polonica, 49 (3): 215-266.

JOHNSON, G. D. 2003. Dentitions of Barbclabornia (newgenus, Chondrichthyes:Xenacanthiformes) from the Upper Palaeozoic of North America. Mitteilungen aus dem Museum für Naturkunde 
in Berlin, Geowissenschaftliche Reihe, 6:125146.

JOHNSON, G. D. 2005a. An unusual tricuspid chondrichthyan tooth from the Lower Permian of Texas, USA. Revista Brasileira de Paleontologia, 8:159-164.

JOHNSON. G. 2005b. Underdeveloped and unusual xenacanth shark teeth from the Lower Permian of Texas. Proceedings of the South Dakota Academy of Science, 84: 215-223.

LONG, J. A. 1995. The Rise of Fishes: 500 Million Years of Evolution. University of New South Wales Press, Sydney, 223p.

MOY-THOMAS, J. A.; R. MILES. 1971. Palaeozoic Fishes. Sauders, Philadelphia, 259p.

MUSSA, D. CARVALHO, R.G.; SANTOS, P.R. 1980. Estudo estratigráfico e Paleoecológico em Ocorrências Fossilíferas da Formação Irati, Estado de São Paulo, Brasil. Boletim do IG/USP, 11: 142-149.

NELSON, J.S. 2006. Fishes of the World. John Wiley \& Sons, New Jersey, 601p.

PARRISH，W. C. 1978. Paleoenvironmental analysis of a Lower Permian bonebed and adjacent sediments, Wichita County,
Texas. Palaeogeography, Palaeoclimatology, Palaeoecology, 24(3): 209-237.

RAGONHA, E.W. 1978. Chondrichthyes do Membro Taquaral (Formação Irati) no Estado de São Paulo. Dissertação de Mestrado, Instituto de Geociências, Universidade de São Pau$10,65 \mathrm{p}$.

RAGONHA, E.W. 1984. Taxonomia de dentes e espinhos isolados de Xenacanthodii (Chondrichthyes, Elasmobranchii) da Formação Corumbataí. Considerações cronológicas e paleoambientais. Instituto de Geociências, Universidade de São Paulo, Tese de Doutoramento, 166p.

RICHTER, M. 2005. A new xenacanthid shark (Chondrichthyes) from the Teresina Formation, Permian of the Paraná Basin, Southern Brazil. Revista Brasileira de Paleontologia, 8(2): 149-158.

SILVA SANTOS, R. 1946. Duas novas formas de elasmobranquios do Paleozóico do Meio Norte do Brasil. Anais da Academia Brasileira de Ciências, 18(4):281-285.

WÜRDIG-MACIEL, N. L. 1975. Ichtiodontes e ichtiodorulitos (Pisces) da Formação Estrada Nova e sua aplicação na estratigrafia do Grupo Passa Dois. Pesquisas, 5:7-165.

Endereço dos autores:

Artur Chahud - Rua Prates, 583, apto 14. Bairro Bom Retiro. São Paulo. SP, CEP 01121- 000. E-mail: arturchahud@yahoo.com

Setembrino Petri - Instituto de Geociências, Departamento de Geologia Sedimentar e Ambiental, Universidade de São Paulo, Rua do Lago, 562, Cidade Universitária CEP 05508-080, São Paulo, SP. E-mail: spetri@usp.br 